\title{
Loss of PTEN function may account for reduced proliferation pathway sensitivity to LY294002 in human prostate and bladder cancer cells
}

Shigeru Kanda $•$ Hiroshi Kanetake • Yasuyoshi Miyata

Shigeru Kanda

Department of Molecular Microbiology and Immunology, Division of Endothelial Cell Biology, Nagasaki University Graduate School of Biomedical Science, 1-7-1 Sakamoto, Nagasaki 852-8501, Japan and National Hospital Organization, Nagasaki Hospital, Sakuragi-machi 41-6, Nagasaki 850-8523, Japan

Hiroshi Kanetake • Yasuyoshi Miyata

Department of Urology, Nagasaki University Graduate School of Biomedical Science, 1-7-1 Sakamoto, Nagasaki 852-8501, Japan

Address for all correspondence:

Shigeru Kanda, M.D., Ph.D.,

National Hospital Organization Nagasaki Hospital

Sakuragi-machi 41-6

Nagasaki 850-8523, Japan

Phone: +81 95-823-2261

Fax: +81 95-828-2616

E-mail: skanda-jua@umin.net 


\begin{abstract}
Purpose: Inhibition of phosphoinositide 3 (PI3)-kinase pathway is attractive for cancer treatment. To examine the role of the phosphatase and tensin homologue (PTEN) in the development of resistance to the treatment.

Methods: We cultured human prostate cancer cells (DU145 and PC-3 cells), and bladder cancer cells (EJ-1 and UM-UC-3 cells) with a PI3-kinase inhibitor, LY294002 for more than 6 weeks and cell proliferation was studied. Activation of Akt1 and ERK was examined by immunoblotting. We introduced the wild type PTEN in UM-UC-3 cells and their prolifartion along with the signaling pathways were also examined.

Results: After 6-weeks, proliferation pathway sensitivity to LY294002 was reduced in cells expressing PTEN, but not in PTEN-null cells. PD98059, a MAPK/ERK kinase inhibitor, significantly inhibited proliferation of PTEN-expressing cells, but not PTEN-null cells. Stable PTEN expression in PTEN-null UM-UC-3 cells increased serum-induced ERK activation and sensitivity to PD98059-treatment, and reduced sensitivity to LY294002 after 6-weeks exposure.

Conclusions: Loss of PTEN function may protect against resistance to PI3-kinase inhibitors through an addiction to the PI3-kinase/Akt pathway.
\end{abstract}

Keywords PI3-kinase, LY294002, resistance, PTEN, ERK 


\section{Introduction}

Protein serine/threonine kinase Akt plays central roles in the phosphoinositide 3-kinase (PI3-kinase)-mediated intracellular signaling. PI3-kinase/Akt pathway is implicated in the proliferation, motility, and survival of tumor cells and is important in angiogenesis (Jiang et al. 2000; Downward 2004; Bader et al. 2005). The endogenous tumor suppressor, phosphatase and tensin homologue (PTEN), dephosphorylates the D3 position of phosphatidylinositol-3,4,5 triphosphate. This antagonizes PI3-kinase-generated second messenger functions and thus inhibits a panel of cellular responses mediated by the PI3-kinase/Akt pathway (Ali et al. 1999; Leslie and Downes 2004; Cully et al. 2006). The loss of functional PTEN is frequently observed in human cancers, including prostate and bladder cancers (Cairns et al. 1997; Aveyard et al 1999). PTEN deficiency is associated with a number of aggressive tumor cell phenotypes and is indicative of a poor prognosis for cancer patients (Ali et al. 1999; Leslie and Downes 2004; Cully et al. 2006).

A potential strategy for treating advanced and/or recurrent human cancers is the use of molecular targeting agents against particular signaling molecules. This strategy may directly inhibit the cellular behaviors of tumor cells and may also indirectly inhibit the progression of tumors in vivo through the blockade of angiogenesis and tumor-stromal interactions. A number of studies have recently shown that cellular loss of functional PTEN led to resistance or reduced sensitivity to molecular targeting compounds, 
irradiation, and chemotherapeutic reagents (Tanaka et al. 2000; Bianco et al. 2003; Nagata et al. 2004; Lee et al. 2005; Jiang et al. 2007). This suggests that the presence of wild type, functional PTEN may benefit therapeutic efficacy in the treatment of human cancers. It has also been shown that loss of functional PTEN increased the sensitivity of pathways involving growth inhibition or induction of apoptosis to acute PI3-kinase inhibition (Zhang et al. 2003; deGraffenried et al. 2004). Although PI3-kinase inhibitors are anticipated to be potent agents in anticancer strategies, the role of PTEN function in chronic PI3-kinase inhibition has not been well elucidated.

In the present study, we investigated the effects of long-term exposure of tumor cells to a PI3-kinase inhibitor, LY294002, on the proliferation of cultured human prostate and bladder cancer cells. We used different cell lines that had different PTEN functionalities to examine whether the expression of wild type PTEN would affect the sensitivity of tumor cells to this compound. We found that expression of wild type PTEN may reduce the sensitivity of pathways involved in proliferation to PI3-kinase inhibition during chronic LY294002 exposure. 


\section{Materials and methods}

Reagents

Anti-PTEN polyclonal antibody was purchased from Upstate Cell Signaling Solutions, Lake Placid, NY. Anti-vinculin monoclonal antibody (clone hVIN-1), minimal essential medium (MEM), non-essential amino acids (NEAA), Ham's F-12 medium, RPMI-1640 medium, and hygromycin were obtained from Sigma Chemical Company, St. Louis, MO. Anti-phospho-Akt1 rabbit monoclonal antibody (pSer473) and anti-phospho-mitogen-activated protein kinase (MAPK) polyclonal antibody (pThr202/Tyr204) were purchased from Cell Signaling Technology, Inc., Beverly, MA. Small molecular weight kinase inhibitors for PI3-kinase and MAPK/extracellular signal-regulated kinase (ERK) kinase (MEK) that directly inhibit their catalytic activities, LY294002, and PD98059, were from Merck Co., Tokyo, Japan. Fetal bovine serum (FBS) and pcDNA3.1/Hygro plasmid were purchased from Invitrogen, Co. Tokyo, Japan. Small, interfering RNA (siRNA) against Human PTEN (HP validated PTEN5) and HiPerFect transfection reagent were purchased from Qiagen Japan, Tokyo, Japan. Anti-ERK1 polyclonal antibody, anti-Akt1 monoclonal antibody, anti-CK2 $\alpha$ polyclonal antibody (C-18), and CK2 $\alpha$ siRNA (a pool of 3 target-specific siRNAs of 20

- 25 nucleotides) were obtained from Santa Cruz Biotechnologies, Santa Cruz, CA. FuGENE 6 reagent was purchased from Roche Diagnostics GmbH, Mannheim, Germany. 
Cell culture

Two human prostate carcinoma cell lines, DU145 and PC-3, obtained from the American Type Culture Collection, were cultured in Ham’s F-12 medium supplemented with 10\% FBS and RPMI-1640 medium supplemented with 10\% FBS, respectively. Two human bladder carcinoma cell lines, EJ-1 (Japanese Cancer Research Resource Bank) and UM-UC-3 (generous gift from Dr. Hiro-omi Kanayama), were cultured in MEM supplemented with NEAA and 10\% FBS. For chronic exposure, cells were cultured in the presence of $10 \mu \mathrm{M}$ LY294002 for more than 6 weeks. We used $10 \mu \mathrm{M}$ LY294002, because this concentration consistently caused statistically significant inhibition of proliferation. The culture medium was refreshed every other day. As described below, cells were examined biologically and biochemically in the presence or absence of LY294002 (short-period treatments). To avoid confusion, parental cells cultured without LY294002 were denoted (pa) cells, and cells chronically exposed to LY294002 were denoted (LY) cells. (LY) cells were cultured with LY294002 in all assays, except when otherwise specified.

Cell proliferation assay

Cells were suspended in medium containing 10\% FBS and seeded into 24-well plates (1 $\times 10^{4}$ cells/well) in the presence of $0.1 \%$ DMSO, LY294002, or PD98059 at the 
indicated concentrations. On the next day, the medium was changed with freshly prepared medium containing either DMSO or inhibitor compounds. On the third day after seeding, cells were detached with trypsin and counted with a hemacytometer.

Examination of labeling index

Labeling indices were examined to determine numbers of cells in S-phase using the Bromodeoxyuridine (BrdU) In-Situ detection kit ${ }^{\circledR}$ (BD Biosciences Pharmingen, San Diego, CA) as described before (Kanda et al. 2005). In brief, cells with either $0.1 \%$ DMSO or $10 \mu \mathrm{M}$ LY294002 were seeded into wells of 48-well-culture plates at a density of $2 \times 10^{4}$ cells/cm². After 2 days, cells were pulse-labeled for 4 h with $10 \mu \mathrm{M}$ of BrdU. Cells were then fixed with the fixation buffer provided in the kit, treated with 70\% ethanol for $20 \mathrm{~min}$, and incubated with $4 \mathrm{M} \mathrm{HCl}$ for $20 \mathrm{~min}$. Cells were washed and incubated with biotinylated anti-BrdU antibody, followed by sequential incubation with streptoavidin-horseradish peroxidase and diaminobenzidine. Finally, cells were counter-stained with hematoxylin and photographs were taken under a light microscope. At least 500 cells were counted for each well, and labeling indices were determined as labeled nuclei/total nuclei ratios and expressed as percentages.

Immunoprecipitation and immunoblotting

Immunoprecipitation and immunoblotting were performed as described previously 
(Kanda et al. 2006b). In brief, cells were incubated in serum-free medium overnight. Cells were stimulated with 10\% FBS for 10 min; controls remained serum-free. Cells were then lysed in Triton X-100 lysis buffer. Akt1 was immunoprecipitated and separated by SDS-polyacrylamide gel electrophoresis (SDS-PAGE). After transfer onto polyvinylidene difluoride membranes, the blots were probed with antibodies, as indicated in the Figures. To determine the expression of PTEN and activation of MAPK/ERK, after stimulation with 10\% FBS as described above, cells were lysed by incubation with boiled SDS-sample buffer followed by the separation with SDS-PAGE, and immunoblotting. To examine the expression of other proteins, blots were stripped as described previously (Kanda et al. 2000), and reprobed with the appropriate antibodies.

Transfection of siRNA

Cells were transfected with siRNA using HiPerFect ${ }^{\circledR}$ reagent as described previously (Kanda et al. 2006a). Briefly, cells were seeded at $2 \times 10^{4}$ cells/well in 24-well plates and cultured overnight. Cells were transfected with the indicated siRNA and cell number was counted 3 days later. Downregulation of target proteins was examined by immunoblotting by the use of total cell lysate (CK2 $\alpha$ and PTEN). To examine the effect of downregulation of PTEN on Akt1 phosphorylation, EJ-1 cells were cultured in $6 \mathrm{~cm}$ dishes, treated with PTEN siRNA, and Akt1 was immunoprecipitated from 95\% of total lysates; downregulation of PTEN expression was examined in the remaining 5\% of total lysate. 
Transfection of wild type PTEN

The plasmid containing the wild type PTEN (Al-Khouri et al. 2005) was a kind gift from Dr. Anna Maria Al-Khouri. The PTEN plasmid comprised the wild type PTEN cDNA subcloned into the pcDNA3.1/Hygro vector. PC-3 cells or UM-UC-3 cells were transfected with a mixture of PTEN plasmid and FuGENE 6 reagent. After 48 h, stably transfected cells were selected with hygromycin. After 2 weeks, hygromycin-resistant clones were picked up and the expression of PTEN was confirmed by immunoblotting. We first transfected the PTEN expression plasmid into PC-3 cells with various amounts of plasmid DNA and three different kinds of liposomes. However, we could obtain only two stable cell lines that expressed a very small amount of wild type PTEN. These cells had a doubling time of more than 6 days (data not shown), and therefore were not adequate for our study. We then attempted to make stable UM-UC-3 cell lines expressing wild type PTEN. Only three hygromycin-resistant clones were obtained after several transfections. Among them, one clone was found to express wild type PTEN at satisfactory levels for our study. We also established a control cell line that contained the empty pcDNA3.1/Hygro vector (Mock transfected cells).

Statistical analysis

Values are presented as mean cell numbers \pm SD. Differences between two groups were 
examined by Mann-Whitney's U test. Differences were considered significant when the $P$-value was less than 0.05 . 


\section{Results}

Chronic treatment with LY294002 effects on the sensitivity of proliferation to this compound in human prostate and bladder cancer cells.

We first examined the expression of PTEN in two human hormone refractory prostate cancer cell lines, DU145 and PC-3. As shown in Fig. 1 A, DU145 cells expressed PTEN, and PC-3 cells did not. We next examined the acute effect of LY294002 on Akt1 phosphorylation at Ser 473 (and subsequent activation). In DU145 cells, Akt1 was phosphorylated by serum treatment and this phosphorylation was blocked by LY294002 (Fig. 1 B). In PC-3 cells, Akt1 was strongly phosphorylated, even in the absence of serum, and LY294002 blocked both basal and serum-induced Akt1 phosphorylation (Fig. 1 B). To examine the effects of chronic LY294002 treatment, we cultured DU145 and PC-3 cells for more than 6 weeks with $10 \mu \mathrm{M}$ LY294002. As shown in Fig. 1 C, serum treatment stimulated Akt1 phosphorylation in untreated DU145 (pa) and PC-3 (pa) cells. Interestingly, serum also induced the phosphorylation of Akt1 in chronically treated DU145 (LY) and PC-3 (LY) cells. This result indicates that after chronic exposure of cells to LY294002, Akt1 could be phosphorylated by external stimuli (serum) even in the presence of LY294002.

We then examined the effect of acute LY294002 treatment on the proliferation of these cells. As shown in Fig. 2 A, LY294002 dose-dependently inhibited the proliferation of DU145 (pa) and PC-3 (pa) cells. The sensitivity of proliferation to LY294002 was 
reduced after chronic LY294002 treatment in DU145 (LY) cells, but not in PC-3 (LY) cells. Labeling index (a percentage of S-phase cells) was also examined. As shown in Fig. 2 B, LY294002 at $10 \mu \mathrm{M}$ significantly inhibited the entry of cells into S-phase by DU145 (pa), PC-3 (pa), and PC-3 (LY) cells, but not by DU145 (LY) cells. Thus, chronic exposure to LY294002 reduced the sensitivity of proliferation to LY294002 in DU145 cells. The IC50 value for LY294002-induced inhibition of cell proliferation was increased 21.4-fold in DU145 (LY) cells compared to DU145 (pa) cells (Table 1). However, the IC50 was similar in PC-3 (LY) and PC-3 (pa) cells.

We also examined the effect of chronic LY294002 exposure on proliferation of bladder cancer cells. Untreated EJ-1 cells expressed PTEN protein, but UM-UC-3 cells did not (Fig. 3 A). To examine whether PTEN expressed in EJ-1 is functionally active, we downregulated the expression of endogenous PTEN by siRNA and examined Akt1 phosphorylation. PTEN siRNA efficiently downregulated the endogenous PTEN in EJ-1 cells. As expected, PTEN siRNA treatment increased basal Akt1 phosphorylation in EJ-1 cells (data not shown), suggesting that PTEN in EJ-1 cells was functionally active. We examined the effect of acute LY294002 treatments on serum-induced phosphorylation of Akt1 in bladder cancer cells. As shown in Fig. 3 B, serum induced the phosphorylation of Akt1 in EJ-1 cells. In UM-UC-3 cells, Akt1 was phosphorylated even in the absence of serum. In both EJ-1 and UM-UC-3 cells, Akt1 phosphorylation was potently inhibited by LY294002 treatments. Next, we examined the effect of chronic LY294002 treatments. After the cultivation of these cells for six weeks in the 
presence or absence of $10 \mu \mathrm{M}$ LY294002, serum-induced phosphorylation of Akt-1 was examined. As shown in Fig. 3 C, in both EJ-1 (LY) and UM-UC-3 (LY) cells, serum was able to induce the phosphorylation of Akt-1 in the presence of LY294002. We also studied the effect of LY294002 on the proliferation of these cells and obtained IC50 values (Table 1). EJ-1 (LY) cells were 13.8-fold more resistant to LY294002-mediated growth inhibition than untreated EJ-1 parental (pa) cells. However, there was no significant difference observed in UM-UC-3 (LY) and (pa) cells.

Because LY294002 can also inhibit the protein kinase, CK2 (Stein 2001), we hypothesized that in DU145 (LY) and EJ-1 (LY) cells, CK2 $\alpha$ (the catalytic subunit of CK2) may participate in the reduced sensitivity of proliferation to LY294002. To investigate this hypothesis, we downregulated the expression of CK2 $\alpha$ by siRNA in DU145 (pa), DU145 (LY), EJ-1 (pa), and EJ-1 (LY) cells, and examined proliferation in the presence or absence of LY294002. As shown in Fig. 4 A, treatment of cells with siRNA for CK2 $\alpha$ efficiently downregulated its expression. In the absence of LY294002, downregulation of CK2 $\alpha$ mildly (but insignificantly) inhibited the proliferation of EJ-1 cells, but not of DU145 cells (Fig. 4 B). However, in the presence of LY294002, downregulation of CK2 $\alpha$ did not inhibit the proliferation of either DU145 (LY) or EJ-1 (LY) cells.

The MEK inhibitor, PD98059, is more effective on proliferation of tumor cells with PTEN than those without PTEN 
Oncogene addiction is a specific dependence of cancer cell proliferation on a particular signaling pathway (Weinstein 2002; Lim and Counter 2005) Since PC-3 and UM-UC-3 cells did not develop resistance to LY294002 after long-term exposure, proliferation of these cells may be more dependent on the PI3-kinase pathways than on other signaling pathways, such as the ERK/MAPK pathway. To explore a possible PI3-kinase addiction, we treated the cells with an ERK/MAPK kinase (MEK) inhibitor, PD98059, and investigated activation of ERK and cell proliferation. As shown in Fig. 5 A, serum was able to activate ERK by phosphorylation (phosphoMAPK) in all prostate and bladder cancer cells. However, the extent of ERK activation in PC-3 cells and UM-UC-3 cells was less than that in DU145 or EJ-1 cells. PD98059 potently inhibited serum-induced ERK activation in all cells. We next examined the proliferation of these cells in the presence of PD98059. As shown in Fig. 5 B, PD98059 significantly inhibited the proliferation of DU145 and EJ-1 cells, but not PC-3 and UM-UC-3 cells. The results suggest that the loss of functional PTEN protein appears to reduce the sensitivity of cellular proliferation to PD98059 and thus, it may cause addiction to the PI3-kinase pathway.

Expression of wild type PTEN in UM-UC-3 cells reduces sensitivity of proliferation to chronic LY294002 exposure

We next examined the effect of wild type PTEN expression in the resistance of proliferation to LY294002. Because an inducible expression system potentially causes 
growth arrest upon PTEN expression, we established a stable cell line by expressing wild type PTEN in PTEN-null cells. Stable expression of wild type PTEN was maintained in a UM-UC-3 cell line and was denoted PTEN-8 (Fig. 6 A). We next examined the serum-induced activation of ERK and Akt. As shown in Fig. 6 B, serum-induced ERK activation was stronger in PTEN-8 cells than in mock transfected cells (Mock). Conversely, phosphorylation of both basal and serum-activated Akt1 in PTEN-8 cells was lower than that in Mock cells (Fig. 6 C). We also examined the effects of PD98059 and LY294002 on proliferation. As shown in Fig. 6 D, PD98059 more potently inhibited the proliferation of PTEN-8 cells than that of Mock cells. However, LY294002 inhibited proliferation similarly in Mock and PTEN-8 cells. When LY294002 and PD98059 was added simultaneously, inhibition of proliferation by LY294002 was further enhanced by PD98059-treatment in PTEN-8 cells, but not in Mock cells. We assessed the IC50 values of parental, Mock, and PTEN-8 cells after 6 weeks in the presence or absence of $10 \mu \mathrm{M}$ LY294002. As shown in Table 2, expression of wild type PTEN in UM-UC-3 cells significantly reduced the sensitivity of proliferation to LY294002 (4.16-fold). 


\section{Discussion}

Synthetic, small molecular weight PI3-kinase inhibitors could be a potentially effective therapeutic strategy for recurrent and advanced human cancers. However, the efficacy of many anti-tumor approaches, including molecular targeting and chemotherapy, is frequently reduced by the loss or mutation of PTEN in human cancers (Tanaka et al. 2000; Bianco et al. 2003; Nagata et al. 2004; Lee et al. 2005; Jiang et al. 2007). Thus, it is important to know whether chronic inhibition of PI3-kinase would also fail to inhibit the proliferation of tumor cells that do not express wild type PTEN. We found that the proliferation of cells expressing wild type PTEN showed reduced sensitivity to LY294002 during chronic exposure. In contrast, tumor cells that lacked PTEN expression maintained LY294002 sensitivity. In clinical use, therapeutic dosing is calculated to attain optimal concentrations in the blood; the optimal concentration is based on the effective concentration that significantly inhibited proliferation of cultured cells in preclinical investigations. Therefore, we treated the cells with LY294002 at 10 $\mu \mathrm{M}$ instead of gradually increasing the concentration, as is frequently done for creating clones that are highly resistant to particular chemicals. The results suggest that during treatment of human advanced cancers, tumor cells expressing wild type PTEN may become resistant to synthetic small molecular weight PI3-kinase inhibitors. The reduction in sensitivity was only about 10 - 20 fold, estimated by IC50 values. However, in clinical medicine, medication doses are not typically increased more than 10 times because of potential toxicity; generally, patients are given the highest dose that can be 
given without adverse effects. Thus, a 10 - 20 fold reduction in sensitivity to the inhibitor would present a critical problem in the treatment of patients with advanced cancers.

The reduced sensitivity to LY294002 after chronic exposure was independent of the phosphorylation status of Akt1, the key downstream signaling molecule of PI3-kinase, because Akt1 phosphorylation by external stimuli (such as serum) was maintained in the presence of LY294002 in DU145 (LY), PC-3 (LY), EJ-1 (LY), and UM-UC-3 (LY) cells. Similar results were reported in a study that showed the sensitivity of tumor cells to CCI-779, a potent inhibitor of mTOR, was dissociated with its ability to inhibit S6 phosphorylation (Neshat et al. 2001). CCI-779 similarly inhibited the phosphorylation of S6 in both CCI-779-sensitive and -resistant cell lines. Thus, differential sensitivity to LY294002 is not explained by difference in biochemical detection of Akt1 activation in PC-3 (LY) and UM-UC-3 (LY) cells.

CK2 consists of two catalytic subunits ( $\alpha$ and $\alpha^{\prime}$ ) and a regulatory subunit $(\beta)$. Recent studies have suggested that CK2 played an important role in proliferation and survival of tumor cells (Ahmad et al. 2005). Downregulation of CK2 $\alpha$ expression by siRNA or inhibition of its catalytic activity by the expression of dominant negative CK2 $\alpha$ inhibited survival and proliferation of cultured cells (Lebrin et al. 2001; Ahmad et al. 2005); this suggested that the CK2 $\alpha$ subunit plays major roles in cellular behaviors. LY294002 inhibits CK2 and PI3-kinase at similar concentrations (Stein 2001). Thus, 
chronic exposure of cells to LY294002 may affect CK2 activity and its downstream targets. In addition, one function of CK2 is the phosphorylation of the C-terminal tail of PTEN, which stabilizes the PTEN protein (Al-Khouri et al. 2005). Thus, chronic inhibition of CK2 may reduce the abundance of PTEN protein. We first examined the expression of PTEN and CK2 $\alpha$ in DU145 (pa), DU145 (LY), EJ-1 (pa), and EJ-1 (LY) cells and found that neither PTEN nor CK2 $\alpha$ expression was altered by chronic exposure to LY294002 (data not shown). We then examined the effect of CK2 $\alpha$ downregulation on proliferation of these cells. However, downregulation did not affect proliferation, even in the presence of LY294002. These results suggest that the reduced sensitivity of proliferation to LY294002 following chronic exposure did not seem to depend on the CK2 $\alpha$ activity.

Another potential mechanism underlying the decreased sensitivity we observed might be oncogenic addiction. Serum-induced activation of ERK was lower in cells that lacked PTEN functionality (PC-3 and UM-UC-3 cells) than in those that possessed PTEN functionality (DU145 and EJ-1 cells). In addition, the stable expression of PTEN in PTEN-null UM-UC-3 cells reduced Akt1 phosphorylation and increased the degree of serum-induced ERK activation. We used siRNA for PTEN to determine the effect of acute downregulation of PTEN on the sensitivity of proliferation to LY294002. However, proliferation sensitivity to LY294002 was not affected by the acute downregulation of PTEN in DU145 and EJ-1 cells (data not shown). Therefore, the chronic loss of PTEN may cause an addiction to the PI3-kinase-dependent signaling 
pathway, which overcomes other signaling pathways, including ERK. It has previously been shown that the loss of PTEN sensitized tumor cells to acute PI3-kinase inhibition (deGraffenried et al. 2004). Our results emphasize that a loss of PTEN would also be favorable for the clinical use of PI3-kinase inhibitors (chronic administration).

It has been reported that the expression of PTEN strongly inhibited the growth of transfected cells (Furnari et al. 1998; Li and Sun 1998). The expression of wild type PTEN in PTEN-null tumor cells (PTEN-8 cells) did not markedly reduce the sensitivity of proliferation to LY294002. In EJ-1 cells, there was a 13.8-fold reduction in sensitivity after chronic exposure to LY294002, but in PTEN-8 cells there was only a 4.16-fold reduction. The reason for this observation is not clear. One possibility is that PTEN-null cells (parental cells of PTEN-8 cells) may already have an addiction to the PI3-kinase/Akt pathway, and ectopic expression of wild type PTEN (such as in PTEN-8 cells) does not alter the addiction. In this case, PTEN-8 cells may be regulated by different molecular machinery from PTEN-expressing cells (DU-145 and EJ-1 cells). Alternatively, a certain amount of PTEN expression might be required for the reduction in sensitivity. These issues remain to be investigated in the future.

In conclusion, our study suggests that the clinical use of PI3-kinase inhibitors for the treatment of patients with advanced cancers may be compromised by the development of resistance to the inhibitors when tumor cells lose the expression of wild type PTEN. 


\section{Acknowledgements}

We are grateful to Dr. Hiro-omi Kanayama (Department of Urology, The University of Tokushima Graduate School Institute of Health Bioscience, Tokushima, Japan) and Dr. Anna Maria Al-Khouri (The Burnham Institute, La Jolla, California) for the kind gift of UM-UC-3 cells and the wild type PTEN plasmid, respectively. We thank Mr. T. Shimogama for his excellent and outstanding help. We greatly appreciate Dr. Paul Kretchmer for editing of this manuscript. This study was partially supported by a Grant-in-Aid for Scientific Research from the Ministry of Education, Science, Sports, and Culture, Japan. 


\section{References}

Ahmad KA, Wang G, Slaton J. Unger G, Ahmed K (2005) Targeting CK2 for cancer therapy. Anti-Cancer Drug 16:1037-1043

Ali IU, Schriml LM, Dean M (1999) Mutational spectra of PTEN/MMAC1 gene: A tumor suppressor with lipid phosphatase activity. J Natl Cancer Inst 91:1922-1932

Al-Khouri AM, Ma Y, Togo SH, Williams S, Mustelin T (2005) Cooperative phosphorylation of the tumor suppressor phosphatase and tensin homologue (PTEN) by casein kinases and glycogen synthase kinase 3ß. J Biol Chem 280:35195-35202

Aveyard JS, Skilleter A, Habuchi T, Knowles MA (1999) Somatic mutation of PTEN in bladder carcinoma. Br J Cancer 80:904-908

Bader AG, Kang S, Zhao L, Vogt PK (2005) Oncogenic PI3K deregulates transcription and translation. Nat Rev Cancer 5:921-929

Bianco R, Shin I, Ritter CA, Yakes FM, Basso A, Rozen N, Tsurutani J, Dennis PA, Miles GB, Arteaga CL (2003) Loss of PTEN/MMAC1/TEP in EGF receptorexpressing tumor cells counteracts the antitumor action of EGFR tyrosine kinase inhibitors. Oncogene 22:2812-2822

Cairns P, Okami K, Halachmi S, Halachmi N, Esteller M, Herman JG, Jen J, Isaacs WB, Bova GS, Sidransky D (1997) Frequent inactivation of PTEN/MMAC1 in primary prostate cancer. Cancer Res 57:4997-5000

Cully M, You H, Levine AJ, Mak TW (2006) Beyond PTEN mutations: the PI3K 
pathways as an integrator of multiple inputs during tumorigenesis. Nat Rev Cancer 6:184-192

deGraffenried LA, Fulcher L, Friendrichs WE, Grunwals V, Ray RB, Hidalgo M.

(2004) Reduced PTEN expression in breast cancer cells confers susceptibility to inhibitors of the PI3 kinase/Akt pathway. Ann Oncol 15:1510-1516

Downward J (2004) PI3-kinase, Akt and cell survival. Seminar Cell Develop Biol $15: 177-182$

Furnari FB, Huang HJ, Cavenee WK (1998) The phosphoinositol phosphatase activity of PTEN mediates a serum-sensitive G1 growth arrests in glioma cells. Cancer Res 58:5002-5008

Jiang B-H, Zheng JZ, Aoki M, Vogt PK (2000) Phosphatidylinositol 3-kinase signaling mediates angiogenesis and expression of vascular endothelial growth factor in endothelial cells. Proc Natl Acad Sci USA 97:1749-1753

Jiang Z, Pore N, Cerniglia GJ, Mick R, Georgescu MM, Bernhard EJ, Hahn SM, Gupta AK, Maity A (2007) Phosphatase and tensin homologue deficiency in glioblastoma confers resistance to radiation and temozolomide that is reversed by the protease inhibitor nelfinavir. Cancer Res 67:4467-4473

Kanda S, Lerner EC, Tsuda S, Shono T, Kanetake H, Smithgall TE (2000) The nonreceptor protein-tyrosine kinase c-Fes is involved in FGF-2-induced chemotaxis of murine brain capillary endothelial cells. J Biol Chem 275:10105-10111

Kanda S, Miyata Y, Mochizuki Y, Matsuyama T, Kanetake H (2005) Aniopoietin 1 is mitogenic for cultured endothelial cells. Cancer Res 65:6820-6827 
Kanda S, Miyata Y, Kanetake H (2006a) Elevated expression of ERK 2 in human tumor cells chronically treated with PD98059. Biochem Biophys Res Commun 345:14811486

Kanda S, Miyata Y, Kanetake H, Smithgall TE (2006b) Fibroblast growth factor-2 induces the activation of Src through Fes, which regulates focal adhesion disassembly. Exp Cell Res 312:3015-3022

Lebrin F, Chambaz EM, Biabchini L (2001) A role for protein kinase CK2 in cell proliferation: evidence using a kinase-inactive mutant of CK2 catalytic subunit $\alpha$. Oncogene 20:2010-2022

Lee S, Choi EJ, Jin C, Kim DH (2005) Activation of PI3K/Akt pathway by PTEN reduction and PIK3CA mRNA amplification contributes to cisplatin resistance in an ovarian cancer cell line. Gynecol Oncol 97:26-34

Leslie NR, Downes CP (2004) PTEN function: how normal cells control it and tumour cells lose it. Biochem J 382:1-11

Li DM, Sun H (1998) PTEN/MMAC1/TEP1 supresses the tumorigenicity and induces G1 cell cycle arrest in human glioblastoma cells. Proc Natl Acad Sci USA 95:15406-15411

Lim K-H, Counter CM (2005) Reduction in the requirement of oncogenic Ras signaling to activation of PI3K/AKT pathway during tumor maintenance. Cancer Cell 8:381392

Nagata Y, Lan KH, Zhou X, Tan M, Esteva FJ, Sahim AA, Klos KS, Li P, Monia BP, Nguyen NT, Hortobagyi GN, Hung MC, Yu D (2004) PTEN activation contributes 
to tumor inhibition by trastuzumab, and loss of PTEN predicts trastuzumab resistance in patients. Cancer Cell 6:117-127

Neshat MS, Mellinghoff IK, Tran C, Stiles B, Thomas G, Petersen R, Frost P, Gibbons JJ, Wu H, Sawyers CL (2001) Enhanced sensitivity of PTEN-deficient tumors to inhibition of FRAP/mTOR. Proc Natl Acad Sci USA 98:10314-10319

Stein RC (2001) Prospects for phosphoinositide 3-kinase inhibition as a cancer treatment. Endocrine Relate. Cancer 8:237-248

Tanaka M, Koul D, Davies MA, Liebert M, Steck PA, Grossman H (2000) MMAC1/PTEN inhibits cell growth and induces chemosensitivity to doxorubicin in human bladder cancer cells. Oncogene 19:5406-5412

Weinstein IB (2002) Cancer. Addiction to oncogenes-The Achilles heal of cancer. Science 297:63-64

Zhang J, Choi Y, Mavromatis B, Lichtenstein A, Li W (2003) Preferential killing of PTEN-null myelomas by PI3K inhibitors through Akt pathway. Oncogene 22:6289-6295 


\section{Figure Legends}

Fig. 1: LY294002 treatment effects on the Akt-1 activation of human prostate carcinoma cells (A) Expression of PTEN protein in human prostate cancer cell lines, DU145 and PC-3. Proteins were visualized by immunoblotting with the indicated antibodies. Anti-vinculin signals indicate the relative protein content in each lane. Results are representative of two independent experiments. (B) Effect of LY294002 (LY) and fetal bovine serum (FBS)-mediated phosphorylation of Akt1 in prostate cancer cells. Cells were either treated or left untreated with $10 \mu \mathrm{M}$ LY294002 for 30 min, and then were stimulated with FBS. Representative results are shown from two independent experiments. (C) FBS-mediated phosphorylation of Akt1 in untreated parental (pa) and chronically LY294002 treated (LY) prostate cancer cells. In (LY) cells, LY294002 at 10 $\mu \mathrm{M}$ was present during serum starvation and serum-treatment. Results are representative of two independent experiments.

Fig. 2: (A) LY294002 effects on the proliferation of prostate cancer cells. Chronically LY294002 treated (LY) and untreated parental (pa) cells were counted after 3 days incubation in the presence $(+)$ or absence (-) of LY294002 at the indicated concentrations. Data were expressed as means \pm SD of triplicate samples. Reproducible results were obtained from 6 experiments. Significant differences are indicated between the first and the last bars underneath the brackets. $* p<0.05$. (B) LY294002 effects on the labeling index of prostate cancer cells. Cells in S-phase were identified by the 
incorporation of BrdU into nuclei. Labeling index was determined as the labeled nuclei/total nuclei ratio and expressed as a percentage. Significant differences are indicated between the first and the last bars underneath the brackets. $* p<0.05$. Representative results are shown from two independent experiments.

Fig. 3: LY294002 treatment effects on the Akt-1 activation of human bladder cancer cells (A) Expression of PTEN protein in human bladder cancer cell lines EJ-1 and UM-UC-3. Proteins were visualized by immunoblotting with the indicated antibodies. Anti-vinculin signals indicate the relative protein content in each lane. Representative results are shown from two independent experiments. (B) Effect of LY294002 (LY) on FBS-mediated phosphorylation of Akt1 in bladder cancer cells. Proteins were visualized by immunoblotting with the indicated antibodies. Representative results are shown from two independent experiments. (C). FBS-mediated phosphorylation of Akt1 in untreated (pa) and chronically LY294002 treated (LY) bladder cancer cells. In (LY) cells, LY294002 at $10 \mu \mathrm{M}$ was present during serum starvation and serum-treatment. Representative results are shown from two independent experiments.

Fig. 4: Effect of CK2 $\alpha$ siRNA on proliferation of human prostate and bladder cancer cells. (A) CK2 $\alpha$ ablation in chronically LY294002 treated (LY) and untreated parental (pa) prostate cancer (DU145) and bladder cancer (EJ-1) cells treated with either control or CK2 $\alpha$ siRNA for 3 days. Proteins were visualized by immunoblotting with the indicated antibodies. Anti-vinculin signals indicate the relative protein content in each 
lane. Representative results are shown from two independent experiments. (B) Effect of CK2 $\alpha$ siRNA on the proliferation of DU145 and EJ-1 cells. Chronically LY294002 treated (LY) and untreated parental (pa) cells were counted after 3-day incubations in the presence (+) or absence (-) of LY294002 and either control or CK2 $\alpha$ siRNA (50 $n M)$. Data were expressed as means $\pm S D$ of triplicate samples. Representative results are shown from two independent experiments. Significant differences are indicated between the bars under the brackets. $* p<0.05$.

Fig. 5: Effect of PD98059 on MAPK/ERK activation and proliferation of prostate and bladder cancer cells. (A) Effect of PD98059 on fetal bovine serum FBS-mediated phosphorylation of ERK (phosphoMAPK) in prostate (DU145 and PC-3) and bladder (EJ-1 and UM-UC-3) cancer cells. Cells were incubated at the indicated concentration of PD98059 for 3 days. Proteins were visualized by immunoblotting with the indicated antibodies. Representative results are shown from two independent experiments. (B) Effect of PD98059 on proliferation of prostate and bladder cancer cells. After incubation in the indicated concentration of PD98059 for 3 days, cell number was counted. Data were expressed as means \pm SD for triplicate samples. Representative results are shown from two independent experiments. Significant differences are indicated between the bars under the brackets. $* p<0.05$.

Fig. 6: Effects of PTEN expression on ERK and Akt-1 activation and proliferation of human bladder cancer cells. (A) Expression of wild type PTEN (PTEN-8) in human 
bladder cancer cell line, UM-UC-3. EJ-1 cells are shown to indicate endogenous expression levels of PTEN. Mock indicates a transfection of empty vector in UM-UC-3 cells, and shows the absence of endogenous PTEN expression. Proteins were visualized by immunoblotting with the indicated antibodies. Anti-vinculin signals indicate the relative protein content in each lane. Representative results are shown from two independent experiments. (B) FBS-induced activation of ERK (phosphoMAPK) in Mock and PTEN-8 cells. Proteins were visualized by immunoblotting with the indicated antibodies. Anti-vinculin signals indicate the relative protein content in each lane. Representative results are shown from two independent experiments. (C) FBS-induced phosphorylation of Akt1 in Mock and PTEN-8 cells. Proteins were visualized by immunoblotting with the indicated antibodies. Representative results are shown from two independent experiments. (D) Effects of PD98059 and LY294002 on the proliferation of Mock and PTEN-8 cells. Cells were counted after 3-day incubations in the presence (+) or absence (-) of LY294002 and/or PD98059 at the concentrations indicated. Data were expressed as means \pm SD for triplicate samples. Representative results are shown from two independent experiments. Significant differences are indicated between the bars under the brackets. $* p<0.05$. 
Table 1. IC50 values for LY294002 treatment in parental and chronically treated human prostate and bladder cancer cells.

\begin{tabular}{lc}
\hline cells & IC50 values $(\mu \mathrm{M})$ \\
\hline DU145 (pa) cells & $1.36 \pm 0.88$ \\
DU145 (LY) cells & $29.13 \pm 15.2$ \\
& \\
PC-3 (pa) cells & $3.23 \pm 1.58$ \\
PC-3 (LY) cells & $3.70 \pm 2.44$ \\
& \\
EJ-1 (pa) cells & $1.43 \pm 0.63$ \\
EJ-1 (LY) cells & $19.73 \pm 8.34$ \\
& \\
UM-UC-3 (pa) cells & $2.63 \pm 1.91$ \\
UM-UC-3 (LY) cells & $2.90 \pm 2.22$ \\
\hline
\end{tabular}

(LY), cultured in the presence of LY294002; (pa) cultured in the absence of LY294002 Values are expressed as means \pm SD from 3 to 6 independent examinations. 
Table 2. IC50 values of parental, Mock-transfected, and PTEN-expressing cells (PTEN-8 cells) chronically treated with or without LY294002.

\begin{tabular}{lc}
\hline cells & IC50 values $(\mu \mathrm{M})$ \\
\hline & \\
Untransfected UM-UC-3 (pa) cells & $6.03 \pm 2.64$ \\
Untransfected UM-UC-3 (LY) cells & $7.09 \pm 4.14$ \\
& \\
Mock-transfected UM-UC-3 (pa) cells & $4.63 \pm 1.90$ \\
Mock-transfected UM-UC-3 (LY) cells & $5.74 \pm 3.42$ \\
& \\
PTEN-8 (pa) cells & $4.33 \pm 2.65$ \\
PTEN-8 (LY) cells & $18.00 \pm 5.90$ \\
\end{tabular}

(LY) cultured in the presence of LY294002; (pa) cultured in the absence of LY294002. IC50 values are expressed as means \pm SD from 3 independent examinations. 


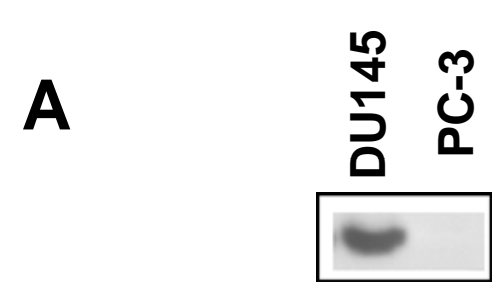

IB : anti-PTEN

IB : anti-vinculin

B

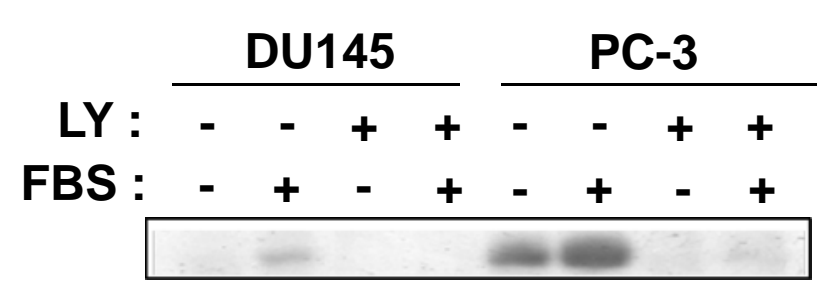

IB : anti-phosphoAkt

IB : anti-Akt1

C

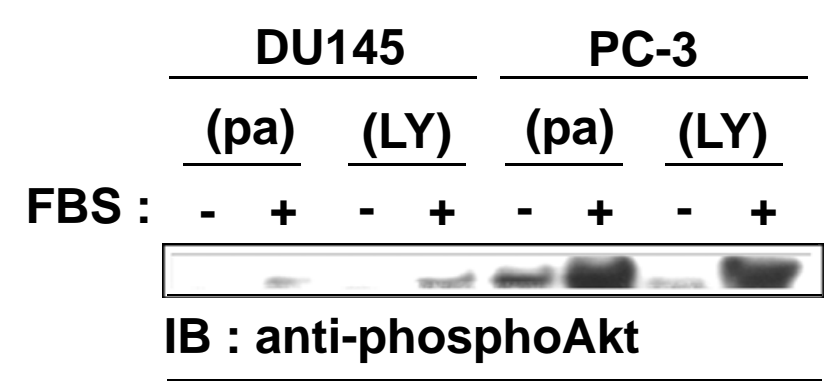

IB : anti-Akt1

Fig. 1. S. Kanda et al. 

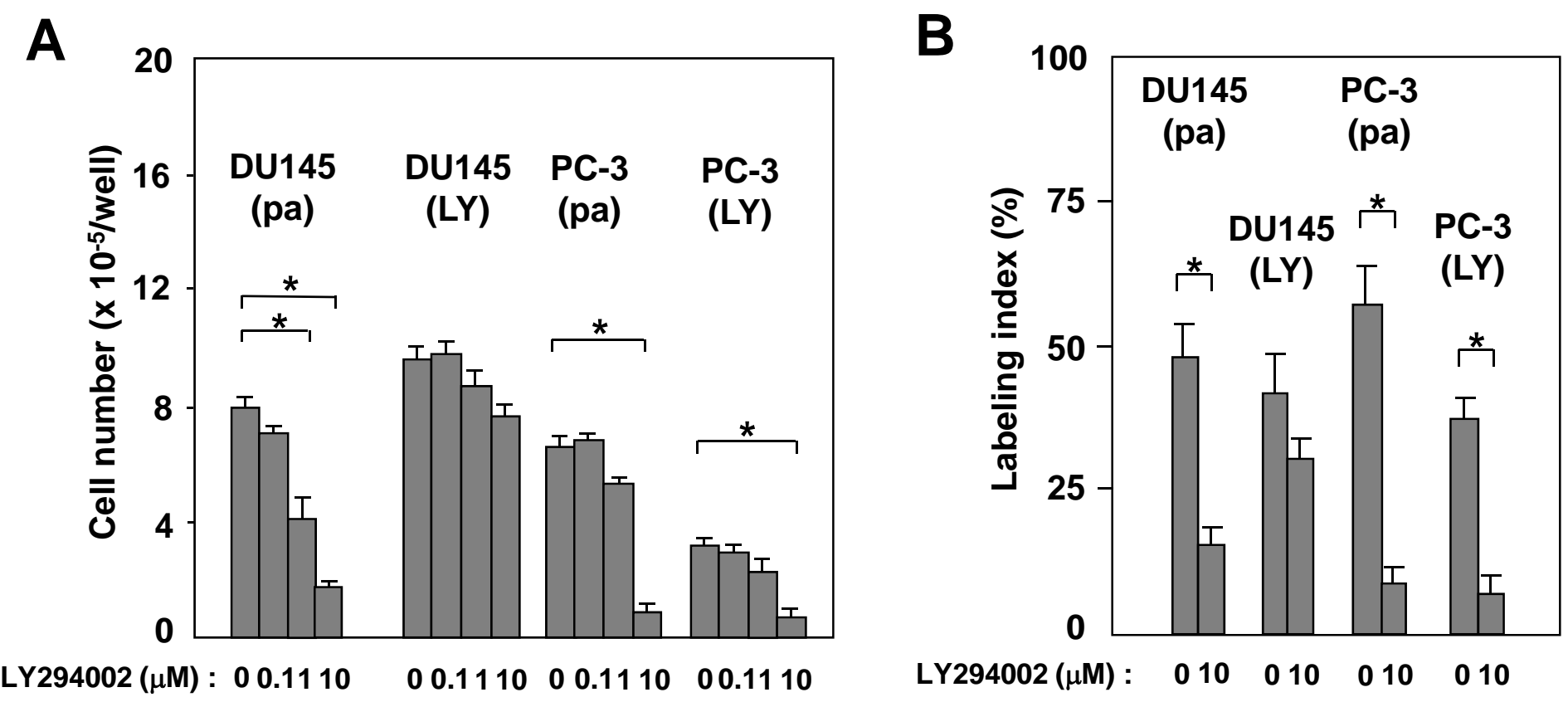

Fig. 2. S. Kanda et al. 


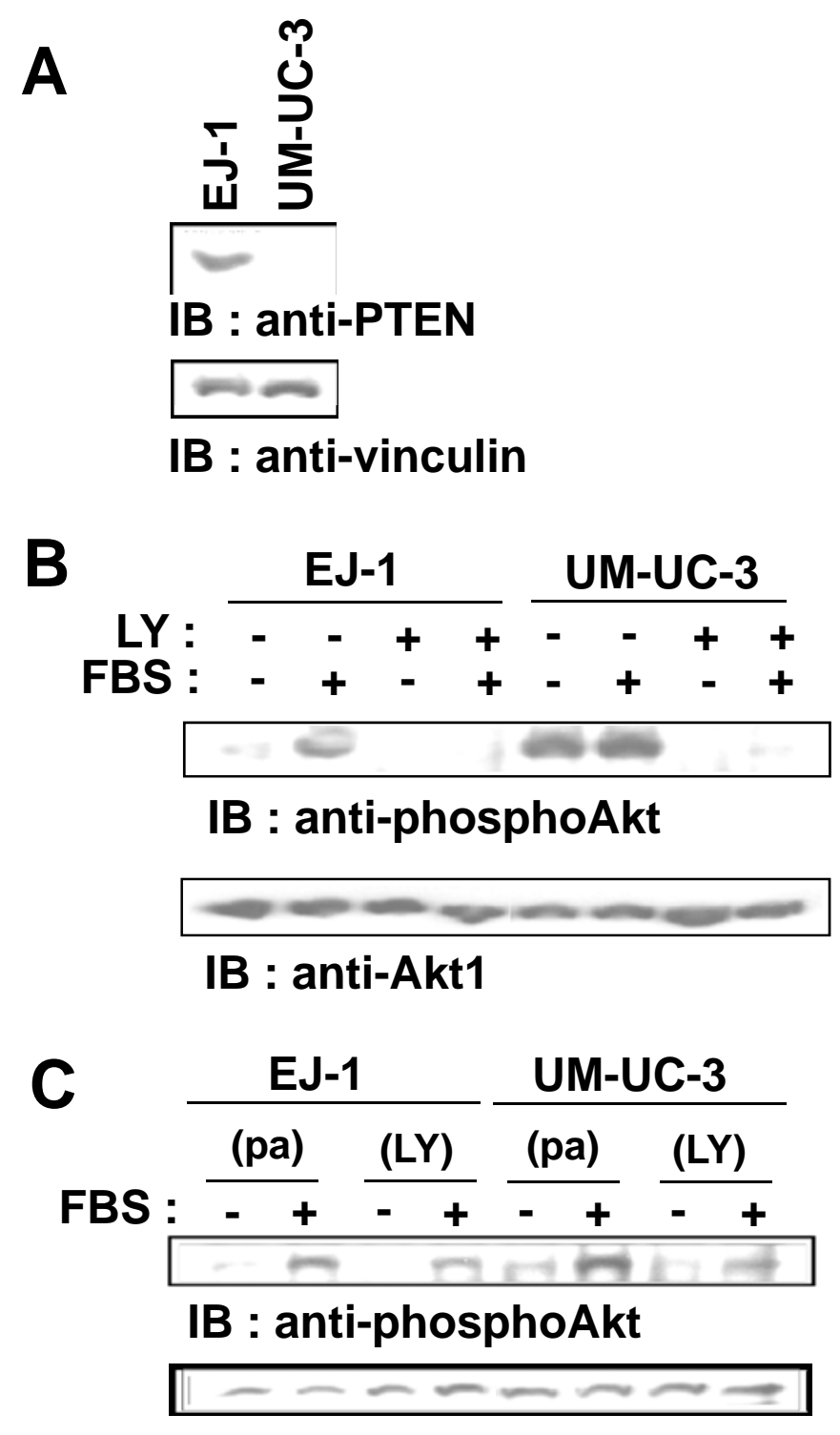

IB : anti-Akt1

Fig. 3. S. Kanda et al. 
A

$\underline{\mathrm{DU}(p a)} \mathrm{DU}(\mathrm{LY}) \mathrm{EJ(pa)} \mathrm{EJ(LY)}$

Control siRNA :

CK2 $\alpha$ SIRNA

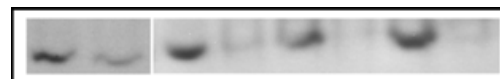

IB : anti- CK2 $\alpha$

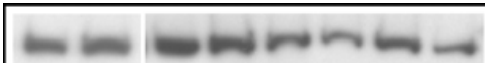

IB : anti-vinculin

B

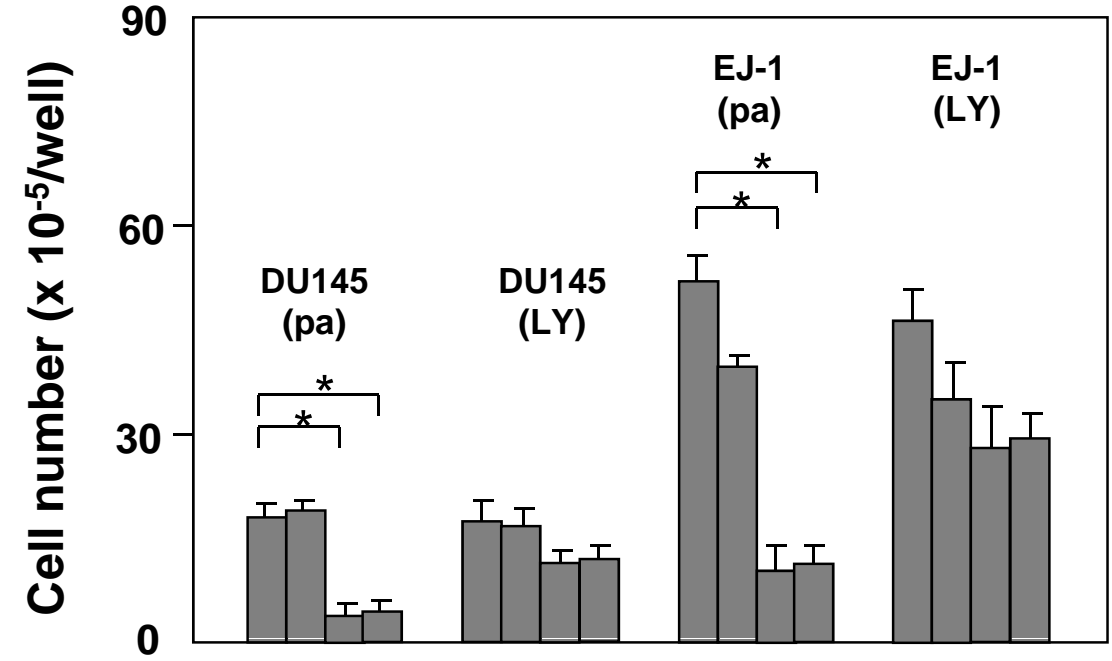

LY294002 :

Control siRNA :

CK2 $\alpha$ SIRNA : $\quad+++$

$-+$

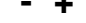

$-\cdots+$

$-++$

Fig. 4. S. Kanda et al. 


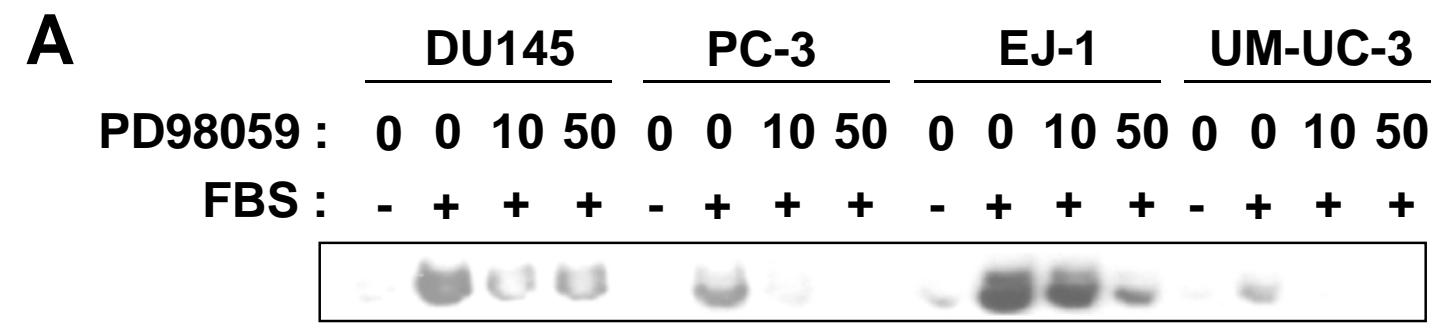

IB : anti-phosphoMAPK

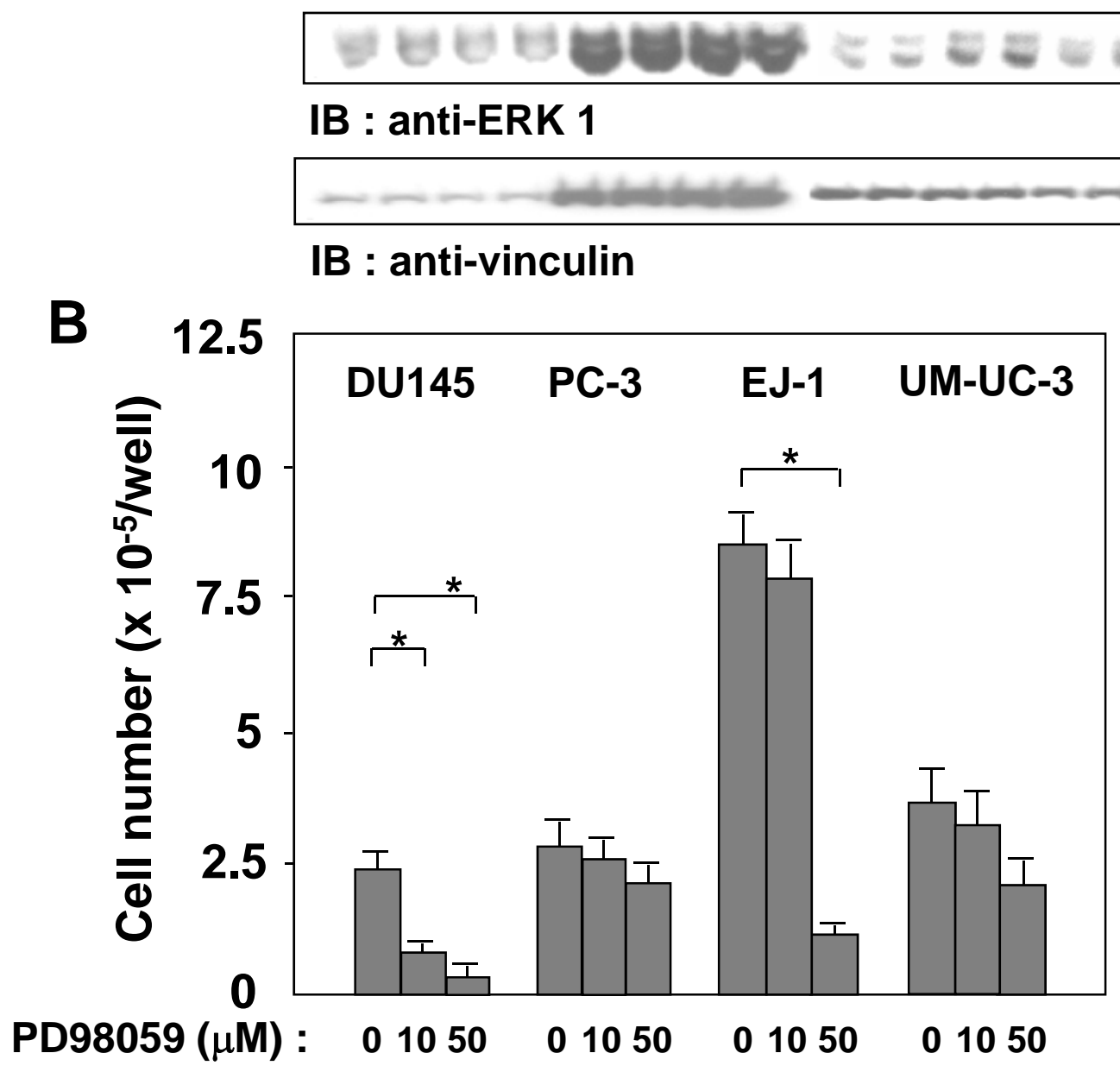

Fig. 5. S. Kanda et al. 


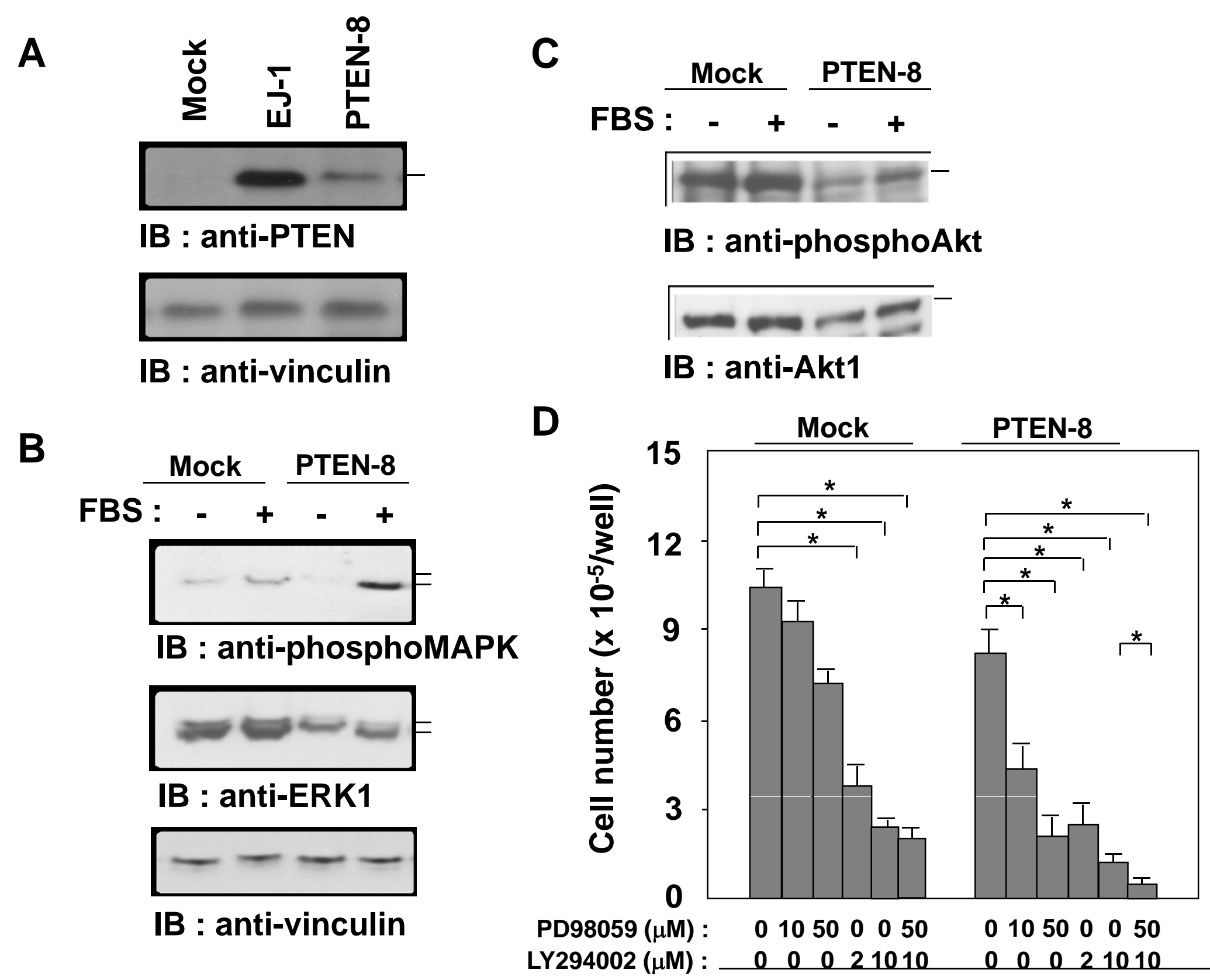

Fig. 6. S. Kanda et al. 